\title{
ON A PROPERTY OF RATIONAL FUNCTIONS. II
}

\author{
Q. I. RAHMAN
}

ABSTRACT. It is shown that if $r_{n}(z)$ is a rational function of degree $n$ such that $r_{n}(0)=1, \lim _{|z| \rightarrow \infty}\left|r_{n}(z)\right|=0$ and all its poles lie in $\left|\zeta_{1}\right| \leqq|z| \leqq 1$ then $\max _{|z|=\rho<\left|\zeta_{1}\right|}\left|r_{n}(z)\right| \geqq 1 /\left(1-\rho^{n}\right)$.

If $r_{n}(z)$ is a rational function of $n$th degree

$$
r_{n}(z)=u(z) / v(z), \quad u, v \text { polynomials }
$$

with

$$
\operatorname{deg} u(z)<\operatorname{deg} v(z)=n, \quad r_{n}(0) \neq 0
$$

then [1] for $1<p \leqq 2$ and arbitrary complex $z_{0}$,

$$
\left\|r_{n}\right\|_{p, z_{0}, \rho}=\left(\frac{1}{2 \pi} \int_{-\pi}^{\pi}\left|r_{n}\left(z_{0}+\rho e^{i \varphi}\right)\right|^{p} d \varphi\right)^{1 / p}
$$

can be estimated nontrivially from below in the maximal regularity circle around $z_{0}$ exclusively by $p,\left|r_{n}\left(z_{0}\right)\right|, \rho, n$ and by the maximum distance of $z_{0}$ from the poles. Here we obtain the sharp lower bound for

$$
\left\|r_{n}\right\|_{\infty, z_{0}, \rho}=\max _{-\pi \leqq \varphi<\pi}\left|r_{n}\left(z_{0}+\rho e^{i \varphi}\right)\right| .
$$

Without loss of generality we may suppose $z_{0}=0, r_{n}(0)=1$; further if the poles are $\zeta_{1}, \zeta_{2}, \cdots, \zeta_{n}$ (repetition permitted), then

$$
0<\left|\zeta_{1}\right| \leqq\left|\zeta_{2}\right| \leqq \cdots \leqq\left|\zeta_{n}\right|=1 \text {, }
$$

and

$$
0<\rho<\left|\zeta_{1}\right| .
$$

THEOREM 1. With the above normalizations we have

$$
\max _{|z|=\rho}\left|r_{n}(z)\right| \geqq \frac{1}{1-\rho^{n}} .
$$

The example $r_{n}^{*}(z)=1 /\left(1-z^{n}\right)$ shows that (7) is sharp.

Received by the editors December 11, 1972.

AMS (MOS) subject classifications (1970). Primary 30A06, 30A40.

Key words and phrases. Rational functions, logarithmic derivative of a polynomial.

(c) American Mathematical Society 1973 
Proof of Theorem 1. Let $\max _{|z|=\rho}\left|r_{n}(z)\right|=M(\rho)$. For every real $\alpha$ the function $u(z)-e^{i \alpha} M(\rho) v(z)$ does not vanish in $|z|<\rho$. Hence if

then

$$
u(z)=1+\sum_{k=1}^{n-1} a_{k} z^{k}, \quad v(z)=1+\sum_{k=1}^{n} b_{k} z^{k},
$$

$$
\begin{aligned}
u(\rho z)-e^{i \alpha} M(\rho) v(\rho z) \equiv & \left(1-e^{i \alpha} M(\rho)\right)+\left(a_{1}-e^{i \alpha} M(\rho) b_{1}\right)(\rho z)+\cdots \\
& +\left(a_{n-1}-e^{i \alpha} M(\rho) b_{n-1}\right)(\rho z)^{n-1}-e^{i \alpha} M(\rho) b_{n}(\rho z)^{n}
\end{aligned}
$$

does not vanish in $|z|<1$. Consequently, the coefficient of $z^{n}$ does not exceed in absolute value the constant term, i.e. $\left|1-e^{i \alpha} M(\rho)\right| \geqq$ $\left|-e^{i \alpha} M(\rho) b_{n} \rho^{n}\right|$. Choosing $\alpha$ appropriately, we get $M(\rho)\left(1-\left|b_{n}\right| \rho^{n}\right) \geqq 1$ or $M(\rho) \geqq 1 /\left(1-\left|b_{n}\right| \rho^{n}\right)$. But $\left|b_{n}\right| \geqq 1$ since the zeros of $v(z)$ lie in $|z| \leqq 1$. Hence $M(\rho) \geqq 1 /\left(1-\rho^{n}\right)$.

The condition (2) is automatically satisfied in the important special case when $r_{n}(z)$ is the logarithmic derivative of a polynomial $\pi_{n}(z)$ of degree $n$ with all zeros in the unit disk; hence Theorem 1 gives a lower bound for $\max _{|z|=\rho}\left|\pi_{n}^{\prime}(z) / \pi_{n}(z)\right|$ depending on $\left|\pi_{n}^{\prime}(0) / \pi_{n}(0)\right|, \rho, n$. However, we show that in this case there is a lower bound which is independent of $\left|\pi_{n}^{\prime}(0) / \pi_{n}(0)\right|$. In fact, we prove

THEOREM 2. Let the zeros $\zeta_{1}, \zeta_{2}, \cdots, \zeta_{n}$ of the polynomial $\pi_{n}(z)$ of degree $n$ be such that $0<\left|\zeta_{1}\right| \leqq\left|\zeta_{2}\right| \leqq \cdots \leqq\left|\zeta_{n}\right| \leqq 1$. Then for $0 \leqq \rho<\left|\zeta_{1}\right|$

$$
\max _{|z|=\rho}\left|\frac{\pi_{n}^{\prime}(z)}{\pi_{n}(z)}\right| \geqq \frac{n \rho^{n-1}}{1-\rho^{n}} .
$$

The example $\pi_{n}^{*}(z)=1-z^{n}$ shows that (8) is sharp.

Proof of TheORem 2. Let $\pi_{n}(z)=\sum_{k=0}^{n} c_{k} z^{k}$ and set

Then

$$
A=\max _{|z|=\rho}\left|\pi_{n}^{\prime}(z) / \pi_{n}(z)\right| \text {. }
$$

$$
\begin{aligned}
& A=\max _{|z|=1}\left|\frac{\pi_{n}^{\prime}(\rho z)}{\pi_{n}(\rho z)}\right|=\max _{|z|=1}\left|\frac{c_{1}+2 c_{2} \rho z+\cdots+n c_{n} \rho^{n-1} z^{n-1}}{c_{0}+c_{1} \rho z+c_{2} \rho^{2} z^{2}+\cdots+c_{n} \rho^{n} z^{n}}\right| \\
& =\max _{|z|=1}\left|\frac{n \bar{c}_{n} \rho^{n-1}+(n-1) \bar{c}_{n-1} \rho^{n-2} z+\cdots+2 \bar{c}_{2} \rho z^{n-2}+\bar{c}_{1} z^{n-1}}{c_{0}+c_{1} \rho z+c_{2} \rho^{2} z^{2}+\cdots+c_{n} \rho^{n} z^{n}}\right| \\
& =n\left|\frac{\bar{c}_{n}}{c_{0}}\right| \rho^{n-1} \max _{|z|=1}\left|\frac{1+\frac{n-1}{n} \frac{\bar{c}_{n-1}}{\bar{c}_{n}} \frac{z}{\rho}+\cdots+\frac{2}{n} \frac{\bar{c}_{2}}{\bar{c}_{n}}\left(\frac{z}{\rho}\right)^{n-2}+\frac{\bar{c}_{1}}{\bar{c}_{n}}\left(\frac{z}{\rho}\right)^{n-1}}{1+\frac{c_{1}}{c_{0}} \rho z+\frac{c_{2}}{c_{0}} \rho^{2} z^{2}+\cdots+\frac{c_{n}}{c_{0}} \rho^{n} z^{n}}\right|
\end{aligned}
$$


$=n\left|\frac{c_{n}}{c_{0}}\right| \rho^{n-1} \max _{|z|=\rho}\left|\frac{1+\frac{n-1}{n} \frac{\bar{c}_{n-1}}{\bar{c}_{n}}\left(\frac{z}{\rho^{2}}\right)+\cdots+\frac{2}{n} \frac{\bar{c}_{2}}{\bar{c}_{n}}\left(\frac{z}{\rho^{2}}\right)^{n-2}+\frac{\bar{c}_{1}}{\bar{c}_{n}}\left(\frac{z}{\rho^{2}}\right)^{n-1}}{1+\frac{c_{1}}{c_{0}} z+\frac{c_{2}}{c_{0}} z^{2}+\cdots+\frac{c_{n}}{c_{0}} z^{n}}\right|$

$\geqq n\left|\frac{c_{n}}{c_{0}}\right| \rho^{n-1} \frac{1}{1-\rho^{n}}$

by Theorem 1. But $\left|c_{n}\right| c_{0} \mid \geqq 1$ since all the zeros of $\pi_{n}(z)$ lie in $z \leqq 1$. Hence $A \geqq n \rho^{n-1} /\left(1-\rho^{n}\right)$.

The problems considered in this paper were suggested to me by Professor Paul Turán for which I am very thankful to him.

\section{REFERENCE}

1. Q. I. Rahman and Paul Turán, On a property of rational functions, Ann. Univ. Sci. Budapest. Eötvös Sect. Math. (to appear).

Department of Mathematics, University of Montreal, Montreal, Quebec, CANADA 